\title{
Fuzzy Fault Tree Analysis for Fault Diagnosis of Cannula Fault in Power Transformer
}

\author{
Sanjay Kumar Tyagi ${ }^{1}$, Diwakar Pandey ${ }^{2}$, Vinesh Kumar ${ }^{2}$ \\ ${ }^{1}$ Department of Applied Mathematics, Amity Institute of Applied Sciences, \\ Amity University, Noida, India \\ ${ }^{2}$ Department of Mathematics, Chaudhary Charan Singh University, Meerut, India \\ E-mail: sanjay_tyagi94@rediffmail.com \\ Received April 17, 2011; revised July 12, 2011; accepted July 19, 2011
}

\begin{abstract}
Being one of the most expensive components of an electrical power plant, the failures of a power transformer can result in serious power system issues. So fault diagnosis for power transformer is highly important to ensure an uninterrupted power supply. Due to information transmission mistakes as well as arisen errors while processing data in surveying and monitoring state information of transformer, uncertain and incomplete information may be produced. Based on these points, this paper presents an intelligent fault diagnosis method of power transformer using fuzzy fault tree analysis (FTA) and beta distribution for failure possibility estimation. By using the technique we proposed herein, the continuous attribute values are transformed into the fuzzy numbers to give a realistic estimate of failure possibility of a basic event in FTA. Further, it explains a new approach based on Euclidean distance between fuzzy numbers, to rank the basic events in accordance with their Fuzzy Importance Index.
\end{abstract}

Keywords: Fault Tree, Power Transformer, Fuzzy Sets, Expert Systems, Fuzzy Importance Index

\section{Introduction}

The involvement of a very large number of variables and their multiple interrelations make the design of a power transformer very complicated. This complicacy in design of a power system and variation in operating conditions causes the occurrence of a fault to be uncertain and random. In present paper we introduce a new approach to the fault detection and analysis of a power transformer.

Fault tree analysis (FTA) is proved to be a very effective tool to predict probability of hazard caused by a sequence and combinations of faults and failure events. A fault tree is a pictorial representation of various combinations of faults leading to hazard. In fault tree analysis, we first explore a hazard and then look for events causing this hazard. In conventional FTA the basic events are assigned a crisp number. But there are various crucial and complex systems of great importance, which imparts vague characteristics. Due to the complexity of systems and their vague nature, it is very difficult to obtain an adequate inference about the failure of these systems.

In 1965, L. A. Zadeh [1] suggested a paradigm shift from a theory of total denial and affirmation to a theory of grading to give the concept of fuzzy set. H. Tanaka, L. T. Fan, F. S. Lai and K. Toguchi [2] used fuzzy set theory to replace a crisp number by fuzzy number for better estimation of failure possibility of top event. D. Singer [3] presented fuzzy set theoretic approach to fault tree analysis. S. Chen [4] used arithmetic of fuzzy numbers to evaluate system reliability. Zong-Xiao Yang, Kazuhiko Suzuki, Yukiyasu Shimada and Hayatoshi Sayama [5] constructed a fuzzy fault diagnostic system, which uses the fuzzy fault tree analysis to represent knowledge of the causal relationships in process operation and control system. Method proposed by him is applied successfully to a nitric acid cooler process plant. Fuzzy set theoretic approach for estimating failure rate parameters developed by D. Pandey and Sanjay Kumar Tyagi [6] provides comprehensive results in estimation of variety of parameters involving human judgment, unreported times, vague operating conditions, etc. D. Pandey and Sanjay Kumar Tyagi [7] developed a technique that has proven successful in other areas of knowledge, fuzzy reasoning, in the evaluation and assessment of equipment failure modes. In this paper probabilistic consideration of basic events is replaced with possibilities, thereby leading to 
fuzzy fault tree analysis.

The failure of power may interrupt various important operations and make a huge damage to the economy of any nation. Power transformer is one of the important electricity equipment used in power networks. Thus the fault diagnosis and its maintenance in a power transformer is the utmost priority of power supply enterprises. Fu Ying-Shun, Liu Fa-Zhan, Zhang Wei-Zheng, Zhang Quing and Zhang Gui-Xin [8] used rough set theory to diagnose faults in a power transformer. Chen Yuliang and Zhang Tiejun [9] also investigated the fuzzy fault tree method in the machinery equipment fault diagnosis. In his theory they used fuzzy mathematics to deal with the uncertainty incurred in the failure probability of basic events. Tong Wu, Guangyu Tu, Z. Q. Bo and A. Klimek [10] also introduced a method of fault diagnosis of power transformer. In their work, they used the analysis method to process the probability of faults without statistical data and developed the methods for the different mode probability data conversion to triangle fuzzy numbers.

Accurate failure statistics is crucial requirement for reliability estimation in power transformer failure. In a situation, wherein failure data may not be corrected accurately due to various reasons, it is more practical to employ linguistic terms to express data value for failure of a particular event. Since a power transformer may be installed under different operating conditions. It seems to be impractical to assign a single fuzzy number to the failure possibility of the basic events in a fault tree analysis. To overcome this problem, in present paper we have categorized the operating condition of a power transformer as "Worst Case Condition", "Conducive Environment" and "Highly Conducive Environment" for a power transformer to work. By "Worst Case Condition" we mean a situation that rarely occurs i.e. in a state of emergency. "Conducive Environment" is a normal state where most of the transformers are installed. Highly Conducive environment is a very special and conducive environment created artificially to keep transformer cool and working for a very long time. Using statistics of failure of power transformer working under different operating conditions each basic event is assigned three fuzzy numbers by Experts A, B and C. But a single fuzzy number is needed for each basic event to evaluate the failure possibility of top event. Sanjay Kumar Tyagi, D. Pandey and Reena Tyagi [11] developed a technique to get a single fuzzy number for each basic event by taking a fuzzy number having least variance with all fuzzy number. But in a natural way, most of the transformers are installed at the places having "Conducive Environmental Conditions". So it is impractical to give equal weightage to the fuzzy numbers assigned by Expert $\mathrm{A}, \mathrm{B}$ and $\mathrm{C}$ to each basic event, which may result in the underestimation/overestimation of failure possibility of the basic events.
Beta distribution is widely used to model probability distributions of variables or project parameters in many areas of operations research like risk analysis for strategic planning, finance and marketing and in decision analysis [12]. Based on beta distribution, here we proposed a very precise and pragmatic approach to estimate the failure possibility of each basic event in fault tree analysis. Three estimates $\tilde{p}_{w}, \quad \tilde{p}_{c}$ and $\tilde{p}_{h}$ suggested by three experts $\mathrm{A}, \mathrm{B}$ and $\mathrm{C}$ are taken into consideration. It is observed that the median (a crisp number) of the triangular fuzzy numbers assigned to three estimates $\tilde{p}_{w}, \tilde{p}_{c}$ and $\tilde{p}_{h}$ are statistically independent. And, since beta distribution is extremely versatile to model variety of uncertainties. So, it is quite useful to apply beta distribution to estimate the parameter "failure possibility" of basic events. In our analysis, we generalize this parameter estimation method by replacing the crisp numbers with fuzzy numbers to obtain the failure possibility of basic events. So it will enable us to give more realistic estimates for failure possibility of basic events.

In FTA the basic events have different importance and improving failure possibility of a basic event having highest importance will improve the reliability of system. H. Furuta and N. Shiraishi [13] proposed the concept of fuzzy importance using max-min fuzzy operator and fuzzy integral. Monte-Carlo simulation is generally used in the determination of importance measure, even though computing process is time consuming. Thus for a very complex system having large number of components, the whole procedure has to be repeated again and again, thus not suitable for the fuzzy approach. P. V. Suresh, A. K. Baber and V. Venkat Raj [14] proposed another method to evaluate an importance measure called fuzzy importance measure (FIM). For effective evaluation of the importance index of each basic events, we have introduced a comparatively easier method to calculate fuzzy importance index (FII), based Euclidean distance between two fuzzy numbers. The FII of different basic events leading Cannula Fault are obtained from the proposed method.

\section{Triangular Fuzzy Numbers and Their Arithmetic}

(a) Triangular Fuzzy Number: A fuzzy number $\tilde{A}$ is termed as triangular fuzzy number if the membership function of fuzzy number $A$ is shown as follows

$$
\mu_{\tilde{A}}(x)= \begin{cases}\frac{x-a_{1}}{a_{2}-a_{1}} & \text { if } a_{1} \leq x \leq a_{2} \\ \frac{a_{3}-x}{a_{3}-a_{2}} & \text { if } a_{2} \leq x \leq a_{3} \\ 0 & \text { otherwise }\end{cases}
$$


In our study, we will use a triplet $\left(a_{1}, a_{2}, a_{3}\right)$ to denote a triangular fuzzy number.

(b) Operations on triangular fuzzy numbers: The addition of triangular fuzzy number $\tilde{A}=\left(a_{1}, a_{2}, a_{3}\right)$ and $\tilde{B}=\left(b_{1}, b_{2}, b_{3}\right)$ is defined as:

$$
\tilde{A}+\tilde{B}=\left(a_{1}+b_{1}, a_{2}+b_{2}, a_{3}+b_{3}\right)
$$

Thus the addition of two triangular fuzzy numbers is again a triangular fuzzy number.

Similarly subtraction of two triangular fuzzy numbers is also a triangular fuzzy number and it can be given by the following expression:

$$
\tilde{A}-\tilde{B}=\left(a_{1}-b_{3}, a_{2}-b_{2}, a_{3}-b_{1}\right)
$$

The multiplication of two fuzzy numbers $\tilde{A}=\left(a_{1}, a_{2}, a_{3}\right)$ and $\tilde{B}=\left(b_{1}, b_{2}, b_{3}\right)$ denoted as $A^{*} B$ can be defined as:

$$
\mu_{\tilde{A} * \tilde{B}}(x)=\left\{\begin{array}{cc}
-D_{1}+\left[D_{1}^{2}+(x-P) / T_{1}\right]^{1 / 2} & P \leq x \leq Q \\
-D_{1}-\left[D_{2}^{2}+(x-R) / U_{1}\right]^{1 / 2} & Q \leq x \leq R \\
0 & \text { otherwise }
\end{array}\right.
$$

where

$$
\begin{gathered}
T_{1}=\left(a_{2}-a_{1}\right)\left(b_{2}-b_{1}\right), \quad T_{2}=a_{1}\left(a_{2}-a_{1}\right)+b_{2}\left(b_{2}-b_{1}\right), \\
U_{1}=\left(a_{2}-a_{1}\right)\left(b_{2}-b_{1}\right), \quad U_{2}=b_{3}\left(a_{2}-a_{1}\right)+a_{3}\left(b_{2}-b_{1}\right), \\
D_{1}=\frac{T_{2}}{2 T_{1}}, \quad D_{2}=-\frac{U_{2}}{2 U_{1}}, \quad P=a_{1} b_{1}, \quad Q=a_{2} b_{2}, \quad R=a_{3} b_{3} .
\end{gathered}
$$

It is evident that the resulting fuzzy number $\tilde{A}^{*} \tilde{B}$ is not a triangular fuzzy number. But in most of the cases, computation with resulting fuzzy numbers becomes very tedious. Thus it is necessary to avoid the second and higher degree terms to make them computationally easy and therefore the product of two fuzzy numbers is reduced to a triangular fuzzy number $(P, Q, R)$ or $\left(a_{1} b_{1}\right.$, $a_{2} b_{2}, a_{3} b_{3}$.

\section{Basics of Possibility Theory}

Using fuzzy set theory, L. A. Zadeh [15] formulated possibility theory in term of fuzzy set. This was an attempt to give a mathematical representation of linguistic uncertainty, i.e. the uncertainty associated with imprecise and vague information. In contrast to the objective character of the probability theory, the possibility theory provides tools for the modeling of subjective probabilities [16]. It is based upon the concept of the possibility distribution. There is a direct connection between possibility and fuzzy sets. This connection can be explained as follows:

Let $X$ denote a variable, taking values from a universal set $R$ and let us consider the equation $X=x$, where $x \in R$ be used to describe the fact that the value of $X$ is $x$.

Now, we consider a fuzzy set $F$ on $R$ that expresses an elastic constraint on values to be assigned to $X$. Then for a particular value $x \in R, F(x)$ gives the degree of compatibility of $x$ with the concept described by $F$. Also for a given proposition $X$ is $F$ based upon fuzzy set $F$, it will be more realistic to interpret $F(x)$ as the degree of possibility that $X=x$. So for a given fuzzy set $F$ on $R$ and the proposition " $X$ is $F$ " the possibility $r_{F}(x)$ of $X=x$ for each $x \in R$ is numerically equal to the degree $F(x)$ to which $x$ belongs to $F$ i.e.

$$
r_{F}(x)=F(x) \text { for all } x \in R \text {. }
$$

The function $r_{F}: X \rightarrow[0,1]$ defined by the equation given above is clearly a possibility distribution function on $R$. For a given $r_{F}$, the associated possibility measure $\left(\operatorname{Pos}_{F}\right)$ is defined for all $A \in P(X)$ by the equation

$$
\operatorname{Pos}_{F}(A)=\sup _{x \in A} r_{F}(x) .
$$

\section{Fuzzy Operators}

Now using algebraic operations on fuzzy numbers (triangular or trapezoidal), we can obtain fuzzy operators corresponding to Boolean operators “AND”, "OR” etc. Let $\tilde{p}_{1}, \tilde{p}_{2} \cdots \tilde{p}_{n}$ are the possibility functions of the basic events $i=1,2, \cdots, n$. Then fuzzy "AND" and "OR" operators denoted by ANF and ORF respectively, can be defined as:

$$
\tilde{p}_{y}=\operatorname{ANF}\left(\tilde{p}_{1}, \tilde{p}_{2}, \cdots, \tilde{p}_{n}\right)=\prod_{i=1}^{n} \tilde{p}_{i}
$$

where $\Pi$ denotes the fuzzy multiplication.

Now let $\tilde{p}_{i}$ 's are represented by triangular fuzzy numbers i.e. $\tilde{p}_{i}=\left(a_{i 1}, a_{i 2}, a_{i 3}\right)$, where $i=1,2, \cdots, n$. Then

$$
\begin{aligned}
& \tilde{p}_{y}=\operatorname{ANF}\left(\tilde{p}_{1}, \tilde{p}_{2}, \cdots, \tilde{p}_{n}\right)=\left(\prod_{i=1}^{n} a_{i 1}, \prod_{i=1}^{n} a_{i 2}, \prod_{i=1}^{n} a_{i 3}\right) \\
& \quad=\left(a_{y 1}, a_{y 2}, a_{y 3}\right), \text { say } \\
& \tilde{p}_{y}=\operatorname{ORF}\left(\tilde{p}_{1}, \tilde{p}_{2}, \cdots, \tilde{p}_{n}\right) \\
& =1-\prod_{i=1}^{n} 1-\tilde{p}_{i}=1-\prod_{i=1}^{n}\left(1-\left(a_{i 1}, a_{i 2}, a_{i 3}\right)\right) \\
& =\left(1-\prod_{i=1}^{n}\left(1-a_{i 1}\right), 1-\prod_{i=1}^{n}\left(1-a_{i 2}\right), 1-\prod_{i=1}^{n}\left(1-a_{i 3}\right)\right) \\
& =\tilde{p}_{y}=\left(a_{y 1}, a_{y 2}, a_{y 3}\right), \text { say. }
\end{aligned}
$$

\section{Proposed Algorithm to Evaluate Failure Possibility of Basic Events}

In this proposed algorithm, fuzzy numbers are used instead of crisp numbers to represent failure probability of 
occurrence of each basic event in fault tree analysis. For the sake of simplicity, triangular fuzzy numbers are used to define the failure possibility of the basic events. Also a triangular fuzzy number is capable to capture the imprecision of experts' assessments, the vagueness of unreliable data and easy to compute.

Step 1: First identify an undesirable top event (Hazard), intermediate events and the basic events leading to top event by exploring history concerned with the failure of that event. And connect these events using logical gates "AND" and "OR" to find the pictorial representation of occurrence of top event.

Step 2: We observe that the basic events are following different statistical property of sampled data collected for a particular event. So the data for the occurrence of the basic events are collected by three experts A, B and $\mathrm{C}$ taking observations under prescribed conditions classified as "Worst Case Condition", "Conducive Environment and "Highly Conducive Environment" respectively.

Step 3: Using sampled data collected by the experts A, $B$ and $C$ the possibility of occurrence of basic events are assigned different fuzzy numbers.

Step 4: It is a well known fact that mostly a system is operated under "Conducive Environment". So it is assumed that the data collected for the failure of a basic event follows a skewed Beta distribution. Thus a technique based on beta distribution is used to find a single fuzzy number to the failure possibility of a basic event. If $\tilde{p}_{w}\left(E_{i}\right), \quad \tilde{p}_{c}\left(E_{i}\right)$ and $\tilde{p}_{h}\left(E_{i}\right)$ are fuzzy numbers assigned to a basic event $E_{i}$ by Expert A, B and C taking observations in "Worst Case Condition", "Conducive Environment" and "Highly Conducive Environment" respectively. Then the failure possibility of the basic event $E_{i}$ may be given as

$$
\tilde{p}\left(E_{i}\right)=\frac{\tilde{p}_{w}\left(E_{i}\right)+4 \tilde{p}_{c}\left(E_{i}\right)+\tilde{p}_{h}\left(E_{i}\right)}{6} .
$$

Step 5: The fuzzy number thus obtained for different basic events are used to compute failure possibility of top event.

\section{Fuzzy Importance Index (FII)}

In fault tree analysis, ranking of basic events as per their importance play a vital role. To improve reliability of a system, it is better to improve the reliability of a basic event, having greater importance instead of the events with less importance. Let $\tilde{p}_{T}$ be the failure possibility of top event and $\tilde{p}_{T_{i}}$ denote the failure possibility of occurrence of top event, if the basic event $E_{i}$ does not happen. In other words we can say $\tilde{p}_{T_{i}}$ be the failure possibility of top event, when failure possibility of basic event $E_{i}$ is a crisp number $\left(\begin{array}{ll}0 & 0\end{array}\right)$. The distance of $\tilde{p}_{T}$ from $\tilde{p}_{T_{i}}$ will determine the importance of a basic event $E_{i}$.

A basic event $E_{i}$ will be of greater importance than the other basic event $E_{j}$, if the distance between $\tilde{p}_{T}$ and $\tilde{p}_{T_{i}}$ is greater than that of $\tilde{p}_{T}$ and $\tilde{p}_{T_{j}}$. The distance between two fuzzy numbers may be obtained by using Hamming or Euclidean distance. The fuzzy importance of each basic event may be quantified as Fuzzy Importance Index (FII).

$$
\operatorname{FII}\left(E_{i}\right)=\text { Distance of } \tilde{p}_{T} \text { from } \tilde{p}_{T_{i}}
$$

If the failure possibilities of the basic events are triangular fuzzy numbers, then the failure possibility of top event will also be a triangular fuzzy numbers. Here we denote $\tilde{p}_{T}$ and $\tilde{p}_{T_{i}}$ by the triplets $(l, m, u)$ and $\left(l^{i}, m^{i}, u^{i}\right)$ respectively. Thus the $\operatorname{FII}\left(E_{i}\right)$ for a basic event $E_{i}$ may be defined as follows.

$$
\begin{aligned}
\operatorname{FII}\left(E_{i}\right) & =\operatorname{ED}\left(\tilde{p}_{T}, \tilde{p}_{T_{i}}\right) \\
& =\sqrt{\left(l-l^{i}\right)^{2}+\left(m-m^{i}\right)^{2}+\left(u-u^{i}\right)^{2}}
\end{aligned}
$$

Thus for basic events $E_{i}$ and $E_{j}$, if $\operatorname{FII}\left(E_{i}\right)>\operatorname{FII}\left(E_{j}\right)$ then the precipitation of basic event $E_{i}$ will be more sensitive than that of event $E_{j}$ to improve system reliability. Using this method we can rank the basic events in accordance with their importance index. And improve the reliability of the system by preventing the failure of a component of greater importance.

\section{Case Study: Failure Possibility of Cannula Fault in Power Transformer}

\subsection{Fault Tree Analysis of Cannula Fault}

The fault tree of Cannula Fault in power transformer is taken as an analytical example to explain the proposed algorithm of fault diagnosis process. The fault tree of Cannula fault in power transformer is shown in Figure 1.

Codes' meaning of basic and intermediate events of Cannula Fault are as follows:

Top event T: Cannula Fault;

Intermediate Events:

$M_{1}$ : Cannula Overheating;

$M_{2}$ : Inside Discharging;

$M_{3}$ : Outer Insulated Flashover;

$M_{4}$ : Deterioration of Insulation;

$M_{5}$ : High Contact Resistance;

$M_{6}$ : Abnormal Overvoltage.

Basic Events:

$E_{1}$ : Over loading;

$E_{2}$ : Natural Aging;

$E_{3}$ : Insulated Damping;

$E_{4}$ : Connector Loosening;

$E_{5}$ : Interface Oxygenating; 


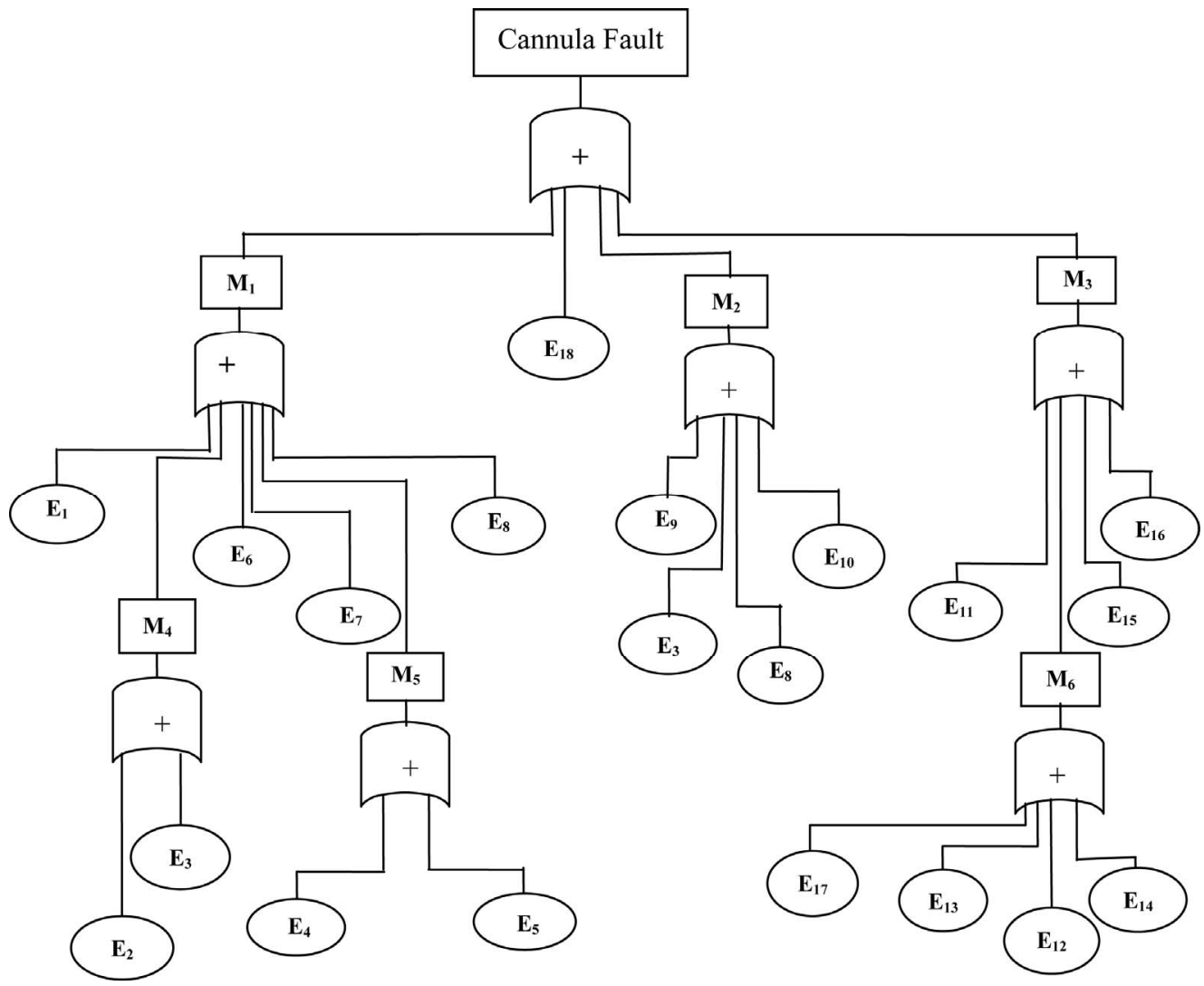

Figure 1. Fault tree of Cannula Fault.

$E_{6}$ : Nicerless Encapsulation;

$E_{7}$ : Outer Short Circuit;

$E_{8}$ : Copper Pole Contact Cable;

$E_{9}$ : Nicerless Dipping;

$E_{10}$ : Unshielded \& imperfect grounding;

$E_{11}$ : Structure unreasonable;

$E_{12}$ : Lightning Conductor Failure;

$E_{13}$ : Near Lightning Spot;

$E_{14}$ : High Energy Lightning;

$E_{15}$ : Annimal;

$E_{16}$ : Dumping Flashover Murry;

$E_{17}$ : Overvoltage by Human Error;

$E_{18}$ : Human Error Fault.

The Boolean expression corresponding to this fault tree can be given as below.

$$
\begin{aligned}
& T=M_{1} \cup E_{18} \cup M_{2} \cup M_{3}, \\
& M_{1}=E_{1} \cup M_{4} \cup E_{6} \cup E_{7} \cup M_{5} \cup E_{8} \\
& M_{2}=E_{9} \cup E_{3} \cup E_{10} \cup E_{8}, \\
& M_{3}=E_{11} \cup M_{6} \cup E_{16} \cup E_{11}, \\
& M_{4}=E_{9} \cup E_{3}, M_{5}=E_{4} \cup E_{5}, \\
& M_{6}=E_{17} \cup M_{13} \cup E_{12} \cup E_{14} .
\end{aligned}
$$

According to the data from reference (Tong $\mathrm{Wu}$, Guangyu Tu, Z. Q. Bo and A. Klimek [10]), the accurate probability value of basic events in fault tree with statistical data is fuzzified and listed in Table $\mathbf{1 .}$

Employing the proposed technique to evaluate the best fuzzy number for failure possibility of each basic event assigned by all three experts, we obtain a unique fuzzy number for each basic event. Fuzzy numbers thus obtained for each basic event are listed in Table 2.

The approximated Fuzzy numbers listed in Table 2 to represent the failure possibility of the basic events are shown in Figure 2.

Using fuzzy operators and triangular fuzzy number approximated to be the possibilities of each basic event, we calculate the possibility of top event. The possibility of top event is resulted as a triangular fuzzy number $(0.862,0.933,0.970)$ expressed as follows and shown in Figure 3.

$$
\mu_{T}(x)= \begin{cases}\frac{x-0.862}{0.071} & \text { if } 0.862 \leq x \leq 0.933 \\ \frac{0.862-x}{0.037} & \text { if } 0.9332 \leq x \leq 0.970\end{cases}
$$

\subsection{Fuzzy Importance Index of Basic Events in Cannula Fault Diagnosis}

To illustrate proposed method of Fuzzy Importance Index (FII), we implement it to the Fault Tree Analysis of 
Table 1. Fuzzy numbers for failure possibility of basic events assigned by experts A, B and C.

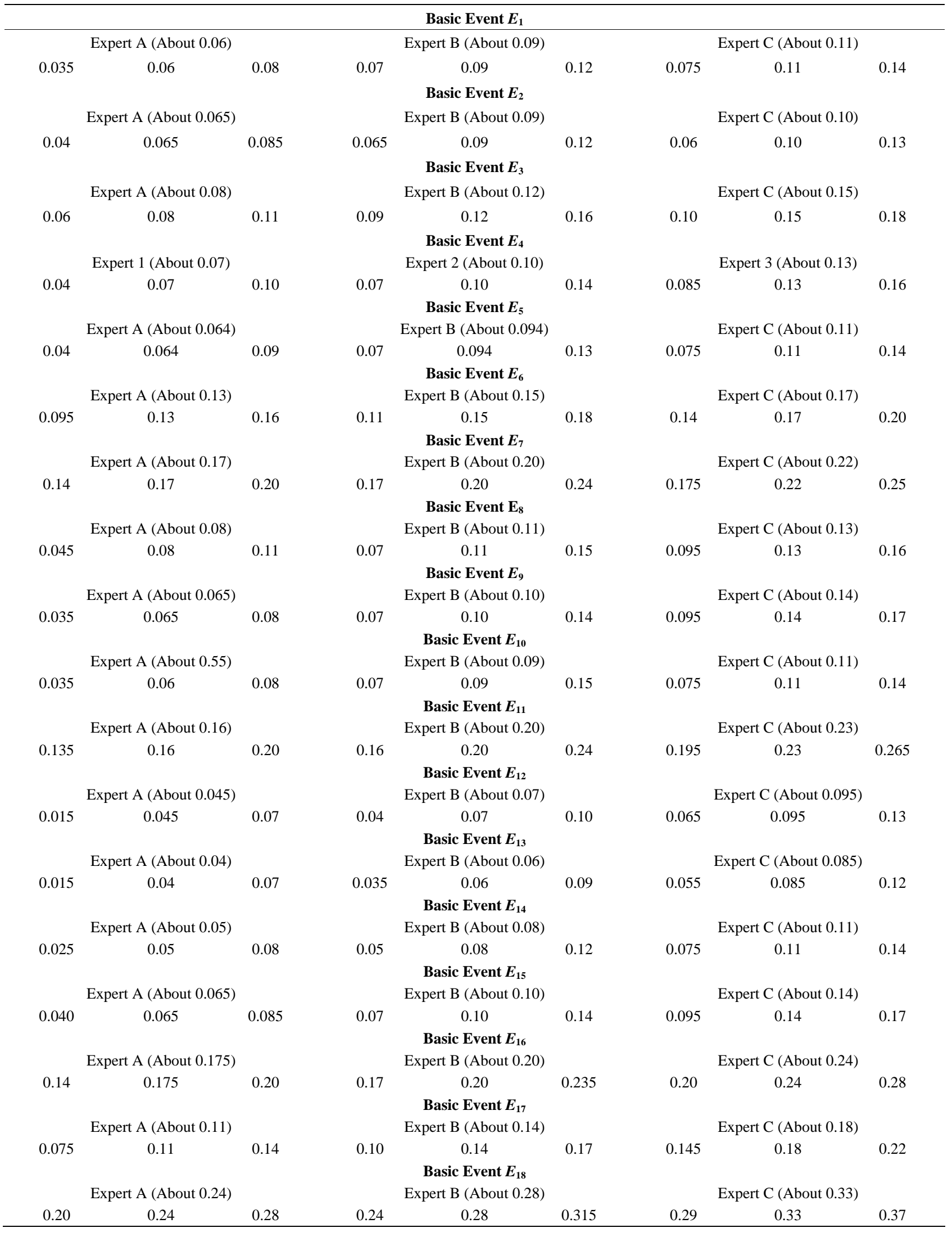


Table 2. Fuzzy numbers approximated for failure possibility of basic events.

\begin{tabular}{|c|c|c|c|c|c|c|c|c|}
\hline \multicolumn{3}{|c|}{ Basic Event $E_{1}$} & \multicolumn{3}{|c|}{ Basic Event $E_{7}$} & \multicolumn{3}{|c|}{ Basic Event $E_{13}$} \\
\hline 0.065 & 0.088 & 0.117 & 0.166 & 0.198 & 0.235 & 0.035 & 0.061 & 0.092 \\
\hline \multicolumn{3}{|c|}{ Basic Event $E_{2}$} & \multicolumn{3}{|c|}{ Basic Event $E_{8}$} & \multicolumn{3}{|c|}{ Basic Event $E_{14}$} \\
\hline 0.06 & 0.088 & 0.116 & 0.07 & 0.108 & 0.145 & 0.05 & 0.08 & 0.117 \\
\hline \multicolumn{3}{|c|}{ Basic Event $E_{3}$} & \multicolumn{3}{|c|}{ Basic Event $E_{9}$} & \multicolumn{3}{|c|}{ Basic Event $E_{15}$} \\
\hline 0.087 & 0.118 & 0.155 & 0.065 & 0.101 & 0.13 & 0.069 & 0.101 & 0.136 \\
\hline \multicolumn{3}{|c|}{ Basic Event $E_{4}$} & \multicolumn{3}{|c|}{ Basic Event $E_{10}$} & \multicolumn{3}{|c|}{ Basic Event $E_{16}$} \\
\hline 0.068 & 0.1 & 0.137 & 0.065 & 0.088 & 0.137 & 0.17 & 0.203 & 0.237 \\
\hline \multicolumn{3}{|c|}{ Basic Event $E_{5}$} & \multicolumn{3}{|c|}{ Basic Event $E_{11}$} & \multicolumn{3}{|c|}{ Basic Event $E_{17}$} \\
\hline 0.066 & 0.092 & 0.125 & 0.162 & 0.198 & 0.238 & 0.102 & 0.142 & 0.173 \\
\hline \multicolumn{3}{|c|}{ Basic Event $E_{6}$} & \multicolumn{3}{|c|}{ Basic Event $E_{12}$} & \multicolumn{3}{|c|}{ Basic Event $E_{18}$} \\
\hline 0.113 & 0.15 & 0.18 & 0.04 & 0.07 & 0.10 & 0.242 & 0.282 & 0.318 \\
\hline
\end{tabular}

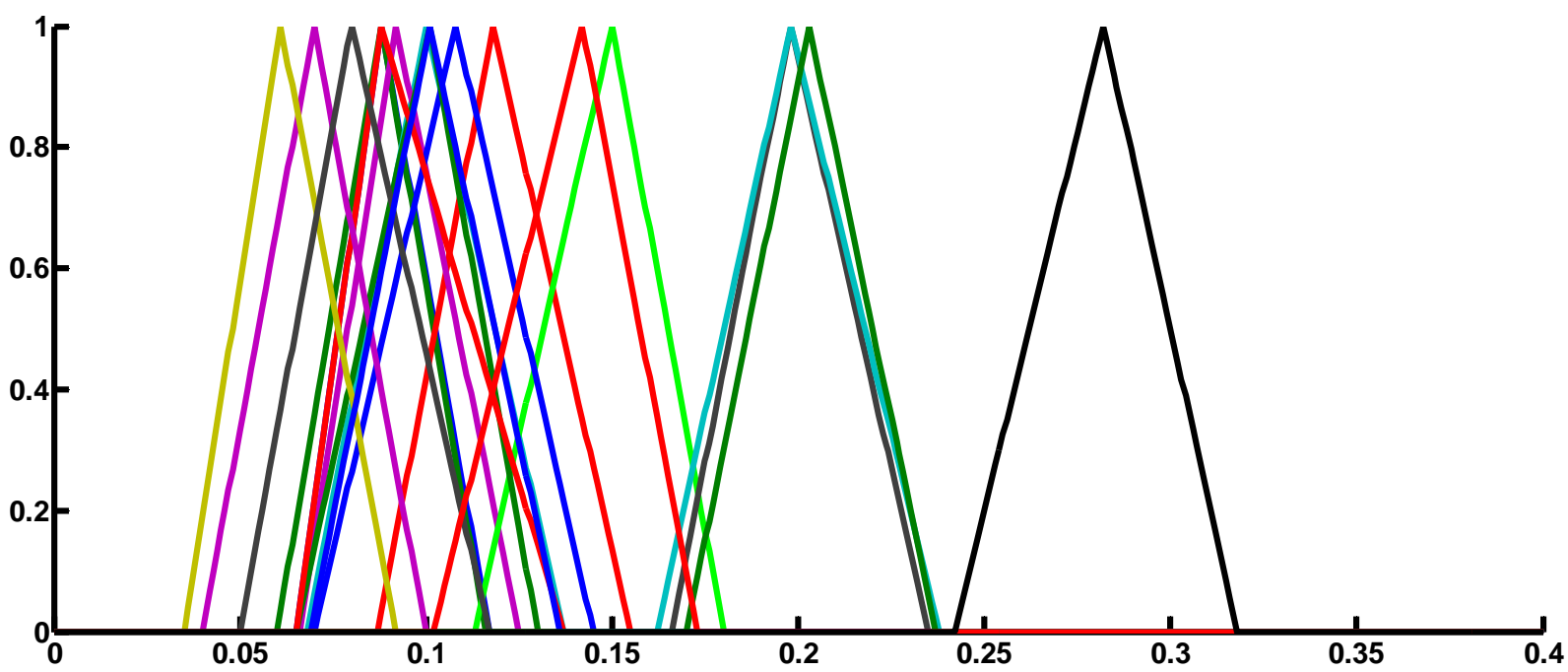

Figure 2. Failure possibilities of basic events.

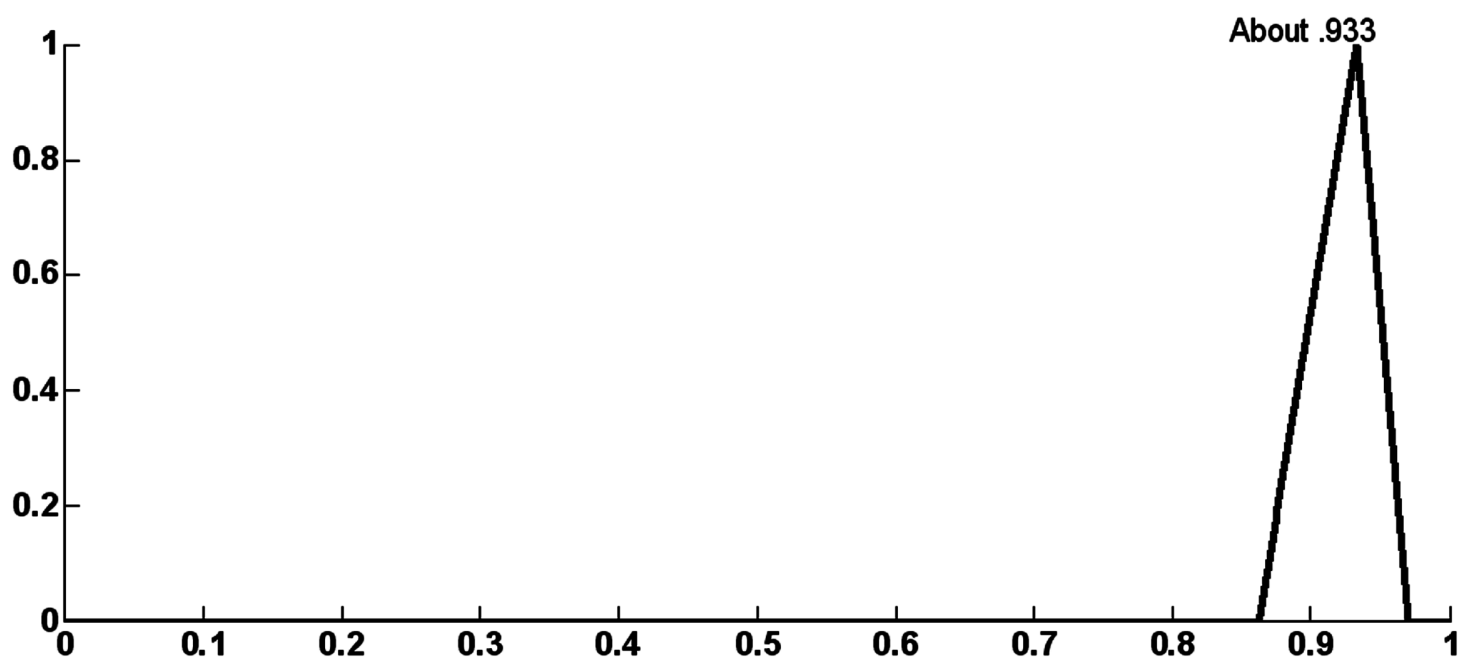

Figure 3. Failure possibility of Cannula Fault. 
Cannula Fault in power transformer. The failure possibility of top event calculated herein is $(0.852,0.933,0.970)$ and denoted as $(l, m, u)$. We use $P_{T_{i}}$ to denote the failure possibility of top event, when basic event $E_{i}$ does not happen. To calculate failure possibility $P_{T_{i}}\left(l^{i}, m^{i}, n^{i}\right)$ the failure possibility for basic event $E_{i}$ is assigned a triangular fuzzy number $(0,0,0)$ i.e. a crisp number zero. The failure possibility $\left(l^{i}, m^{i}, n^{i}\right)$ of top event for each basic event $E_{i}$, thus obtained is listed in Table 3.
The fuzzy importance index for each basic event $E_{i}$, obtained by using the following expression is listed in Table 4 and shown in Figure 4.

$$
\operatorname{FII}(i)=\sqrt{\left(l-l^{i}\right)^{2}+\left(m-m^{i}\right)^{2}+\left(u-u^{i}\right)^{2}}
$$

In Figure 4 the height of the columns represents the FII of each basic event. Here we see that the columns are of varying height, proving that the basic events possess different FII.

Table 3. Possibility of top event when basic event $E_{i}$ does not happen.

\begin{tabular}{cc}
\hline Basic Event $\left(\boldsymbol{E}_{\boldsymbol{i}}\right)$ & Possibility of top event when basic event $\boldsymbol{E}_{\boldsymbol{i}}$ does not happen $\left(P_{T_{\boldsymbol{i}}}\right)$ \\
\hline$E_{1}$ & $(0.852,0.927,0.966)$ \\
$E_{2}$ & $(0.852,0.927,0.966)$ \\
$E_{3}$ & $(0.834,0.914,0.958)$ \\
$E_{4}$ & $(0.852,0.926,0.966)$ \\
$E_{5}$ & $(0.852,0.926,0.966)$ \\
$E_{6}$ & $(0.844,0.921,0.964)$ \\
$E_{7}$ & $(0.834,0.917,0.961)$ \\
$E_{8}$ & $(0.840,0.916,0.959)$ \\
$E_{9}$ & $(0.852,0.926,0.966)$ \\
$E_{10}$ & $(0.852,0.0927,0.966)$ \\
$E_{11}$ & $(0.835,0.917,0.961)$ \\
$E_{12}$ & $(0.856,0.928,0.967)$ \\
$E_{13}$ & $(0.857,0.929,0.967)$ \\
$E_{14}$ & $(0.854,0.927,0.966)$ \\
$E_{15}$ & $(0.851,0.926,0.966)$ \\
$E_{16}$ & $(0.833,0.916,0.961)$ \\
$E_{17}$ & $(0.846,0.922,0.964)$ \\
$E_{18}$ & $(0.817,0.907,0.957)$ \\
\hline
\end{tabular}

Table 4. Fuzzy importance index of basic events.

\begin{tabular}{cc}
\hline Basic Event $\left(\boldsymbol{E}_{\boldsymbol{i}}\right)$ & Fuzzy Importance Index (FII) \\
\hline$E_{13}$ & 0.005 \\
$E_{12}$ & 0.006 \\
$E_{14}$ & 0.008 \\
$E_{1}$ & 0.010 \\
$E_{2}$ & 0.010 \\
$E_{4}$ & 0.010 \\
$E_{5}$ & 0.010 \\
$E_{9}$ & 0.010 \\
$E_{10}$ & 0.010 \\
$E_{15}$ & 0.011 \\
$E_{17}$ & 0.017 \\
$E_{6}$ & 0.019 \\
$E_{8}$ & 0.026 \\
$E_{11}$ & 0.029 \\
$E_{7}$ & 0.030 \\
$E_{16}$ & 0.031 \\
$E_{3}$ & 0.032 \\
$E_{18}$ & 0.050 \\
\hline
\end{tabular}




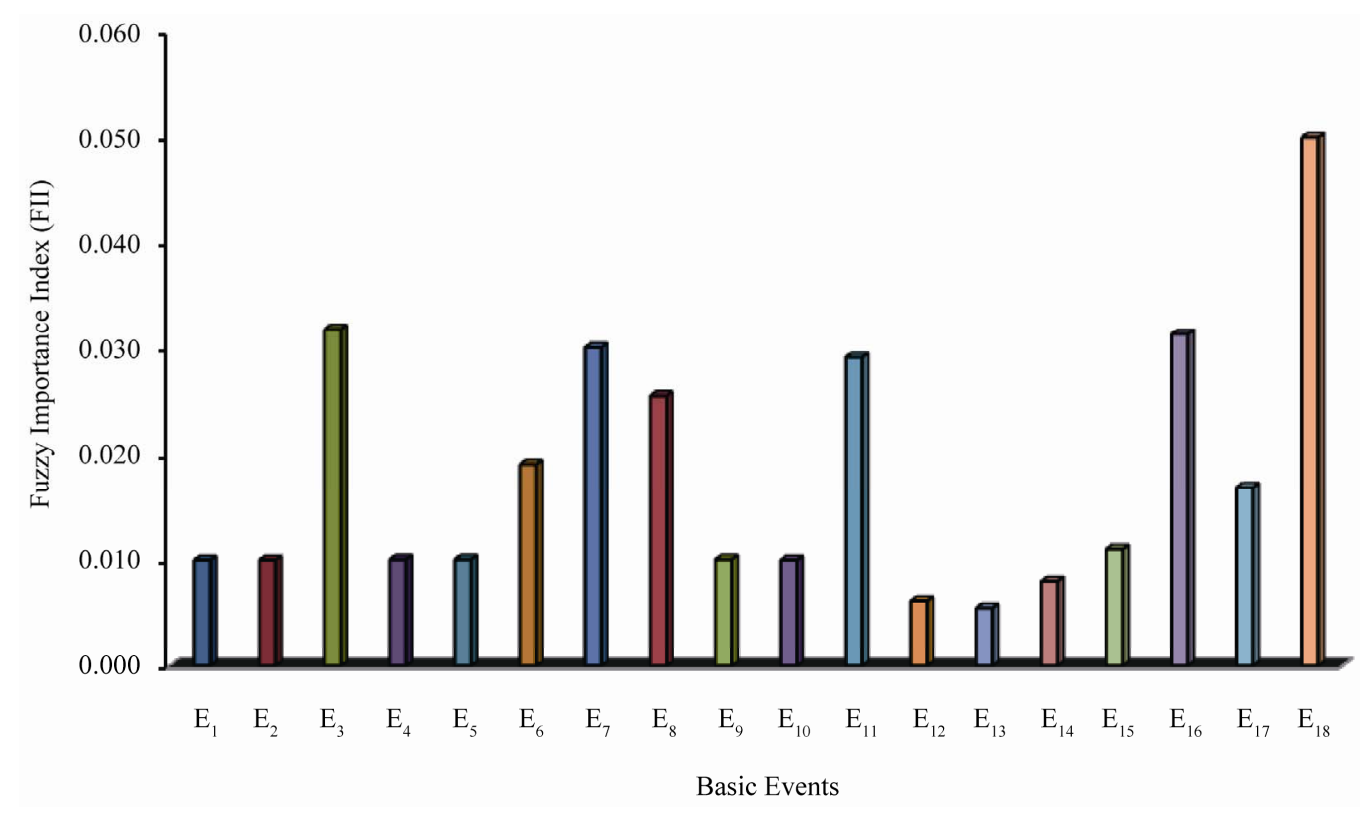

Figure 4. Fuzzy Importance Index (FII) of basic events.

\section{Conclusions and Discussion of Result}

In present paper, we introduce a novel approach to approximate the failure possibility of basic events, if more than one fuzzy number is assigned to a particular basic event by different experts. The possibilities of basic events are considered to be triangular fuzzy numbers. Three fuzzy numbers are assigned to each basic event by the Experts A, B and C. These experts collect data of failure for each component in three different operating conditions "Worst Case Condition", "Conducive Environment" and "Highly Conducive Environment". Unlike previous techniques, we investigate the operating conditions rigorously and assess the weightage of each of them. Taking view of this, we use the parameter estimation method used in PERT method and generalize it by replacing crisp numbers with fuzzy numbers, to obtain most likely fuzzy number to represent the failure possibility of basic events. The proposed method seems to be very pragmatic and preclude underestimation/overestimation of failure possibility for basic events.

Also it is a well known fact that all basic events do not contribute equally in failure of a system i.e. the occurrence of top event. Thus it is important to assess the importance of each basic event. Herein we employ a very effective and computationally easy technique based on Euclidean Distance between fuzzy numbers.

The proposed methods are implemented to the Cannula Fault in Power Transformer. We have eighteen basic events leading the occurrence of top event. Using proposed technique to calculate FII of basic events, we list them in Table 4 in ascending order of their FII and put in Figure 4. We conclude that the basic event $E_{18}$ having highest FII, causes precipitation of system more rapidly than the basic event $E_{13}$ with least FII. Therefore the reliability of Cannula and hence of Power Transformer may be improved by preventing occurrence of basic event $E_{18}$.

\section{Acknowledgements}

The authors are grateful to the anonymous reviewers for their critical evaluation and constructive suggestions.

\section{References}

[1] L. A. Zadeh, "Fuzzy Sets,” Information and Control, Vol. 8, No. 3, 1965, pp. 338-353. doi:10.1016/S0019-9958(65)90241-X

[2] H. Tanaka, L. T. Fan, F. S. Lai and K. Toguchi, "FaultTree Analysis by Fuzzy Probability,” IEEE Transactions on Reliability, Vol. 32, No. 5, 1983, pp. 453-457. doi:10.1109/TR.1983.5221727

[3] D. Singer, "A Fuzzy Set Approach to Fault Tree Analysis,” Fuzzy Sets and Systems, Vol. 34, No. 2, 1990, pp. 145-155. doi:10.1016/0165-0114(90)90154-X

[4] S. Chen, "Fuzzy System Reliability Analysis Using Fuzzy Number Arithmetic Operations,” Fuzzy Sets and Systems, Vol. 64, No. 1, 1994, pp. 31-38. doi:10.1016/0165-0114(94)90004-3

[5] Z.-X. Yang, K. Suzuki, Y. Shimada and H. Sayama, "Fuzzy Fault Diagnostic System Based on Fault Tree Analysis," Proceedings of the International Joint Conference of the Fourth IEEE International Conference on Fuzzy Systems and The Second International Fuzzy Engineering 
Symposium, Yakohama, 20-24 March 1995, Vol. 1, pp. 165-170. doi:10.1109/FUZZY.1995.409676

[6] D. Pandey and S. K. Tyagi, "Profust Reliability of a Gracefully Degradable System,” Fuzzy Sets and Systems, Vol. 158, No. 7, 2007, pp. 794-803. doi:10.1016/j.fss.2006.10.022

[7] D. Pandey, S. K. Tyagi and V. Kumar, "Failure Mode Screening Using Fuzzy Set Theory,” International Mathematical Forum, Vol. 4, No. 16, 2009, pp. 779-794

[8] Y.-S. Fu, F.-Z. Liu, W.-Z. Zhang, Q.-I. Zhang and G.-X. Zhang, "The Fault Diagnosis of Power Transformer Based on Rough Set Theory," Proceedings of the China International Conference on Electricity Distribution, Guangzhou, 10-13 December 2008, pp. 1-5.

[9] Y. L. Chen and T. J. Zhang, "Research on Application of Fuzzy Fault Tree Analysis Method in the Machinery Equipment Fault Diagnosis," Proceedings of 2nd International Conference on Informatics in Control, Automation and Robotics, Wuhan, 6-7 March 2010, pp. 84-87.

[10] T. Wu, G. Y. Tu, Z. Q. Bo and A. Klimek "Fuzzy Set Theory and Fault Tree Analysis Based Method Suitable for Fault Diagnosis of Power Transformer," Proceedings of the 14th International Conference on the Intelligent
System Applications to Power Systems, Kaohsiung, 5-8 November 2007, pp. 487-491. doi:10.1109/ISAP.2007.4441664

[11] S. K. Tyagi, D. Pandey and R. Tyagi, "Fuzzy Set Theoretic Approach to Fault Tree Analysis," International Journal of Engineering, Science and Technology, Vol. 2, No. 5, 2010, pp. 276-283.

[12] S. D. Moitra, "Skewness and the Beta Distribution,” Journal of Operational Research Society, Vol. 41, No. 10, 1990, pp. 953-961.

[13] H. Furuta and N. Shiraishi, "Fuzzy Importance in Fault Tree Analysis,” Fuzzy Sets and Systems, Vol. 12, No. 3, 1984, pp. 205-213. doi:10.1016/0165-0114(84)90068-X

[14] P. V. Suresh, A. K. Babar and V. V. Raj, "Uncertainty in Fault Tree Analysis: A Fuzzy Approach,” Fuzzy Sets and Systems, Vol. 83, No. 2, 1996, pp. 135-141. doi:10.1016/0165-0114(95)00386-X

[15] L. A. Zadeh "Fuzzy Sets as the Basis for a Theory of Possibility,” Fuzzy Sets and Systems, Vol. 1, No. 1, 1978, pp. 3-28. doi:10.1016/0165-0114(78)90029-5

[16] G. J. Klir and B. Yuan, "Fuzzy Sets and Fuzzy Logic: Theory and Applications,” Prentice-Hall, Upper Saddle River, 1995. 